\title{
Nuclear structure aspects via g-factor measurements: pushing the frontiers
}

\author{
Diego A. Torres*, F. Ramirez \\ Departamento de Física, Universidad Nacional de Colombia, Colombia \\ E-mail: datorresgeunal.edu.co
}

\begin{abstract}
During the past decades systematic studies of the magnetic moments of the $2_{1}^{+}$states in eveneven nuclei have been performed by several groups around the globe, providing valuable tests of theoretical models of nuclear structure. Such measurements have unveiled important features of the interplay between single-particle and collective excitation degrees of freedom. It is widely accepted that the possibility to distinguish between single-particle and collective behavior in nuclear states, thanks to the microscopic description of the states under study, is one of the greatest achievements of the research in nuclear magnetic moments. Experimentally, much progress has been achieved by using the transient field technique in inverse kinematics. However, challenges arise from the difficulty to extend these measurements to $4_{1}^{+}, 2_{2}^{+}$, and higher excited states, the use of low intensity radioactive beams, and reactions such as $\alpha$ transfer and fusion-evaporation. In this contribution an overview of the trend of magnetic moments ( $g$ factors) for several isotopic chains, measured using the transient field technique, will be presented. The results are evaluated in the context of the systematic of $g$ factors in several mass regions. Some of the challenges and frontiers of future $g$-factor measurements will be discussed.
\end{abstract}

X Latin American Symposium on Nuclear Physics and Applications (X LASNPA),

1-6 December 2013

Montevideo, Uruguay

${ }^{*}$ Speaker. 


\section{Introduction}

During the last decades a wealth of experimental and theoretical information has been obtained in the field of nuclear structure with the help of the study of nuclear magnetic moments of excited states $[1,2,3,4]$. The outstanding property of the bare-spin $g$ factors being positive for protons, $g_{s}^{\pi}=+5.586$, and negative for neutrons, $g_{s}^{\mu}=-3.826$, enable the determination of the proton and neutron contributions to the wave function of individual states, in particular for those nuclei near closed shells. It is worth to notice that most of the $g\left(2_{1}^{+}\right)$factors are close to the collective value $g=Z / A$. The study of the deviations from this collective value provides key information about the evolution of the nuclear structure along several isotopic chains, and is presented below.

Recently, the measurements have been concentrated on the systematic study of even-even nuclei, specifically states with $J^{\pi} \geq 2^{+}$, with a special focus on the improvement of the accuracy of the measurements $[5,6]$. In addition to the latter, the implementation of radioactive beam facilities has extended our knowledge to regions away from stability $[7,8,9,10]$. Another successful technique to reach radioactive species has been the use of $\alpha$-transfer reactions from stable beams $[11,12,13,14,15]$.

On the theoretical side, Large Scale Shell Model calculations (LSSM) using codes such as ANTOINE [16] and the Oslo code [17], in combination with realistic interactions capable to describe large model spaces $[18,19,20]$, have given us the capability to understand the microscopic behavior of nuclei in several mass regions.

In this contribution, results on nuclear magnetic moments, using the Transient Field (TF) technique for several isotopic chains, will be reviewed. The challenges and difficulties for future uses of the TF technique with radioactive species and different reactions will be addressed.

\section{The region between $N=20$ and $N=28$}

$$
\mathrm{g}\left(2_{1}^{+}\right) \text {factors }
$$

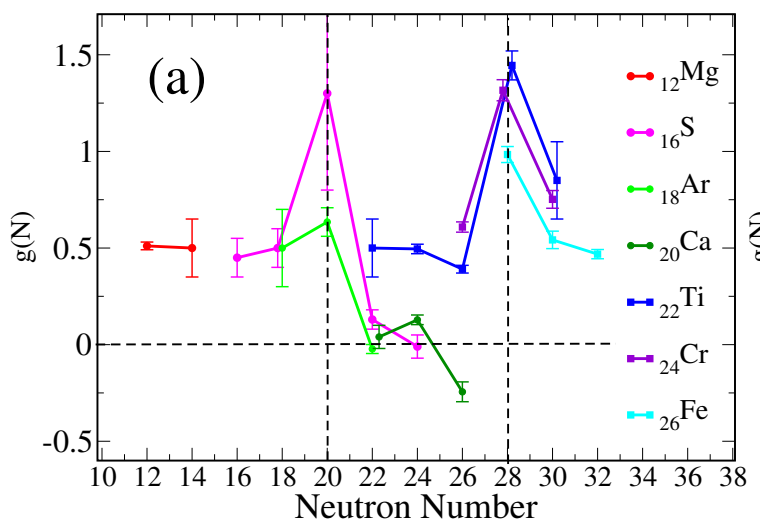

$\mathrm{g}\left(2^{+}\right)$factors

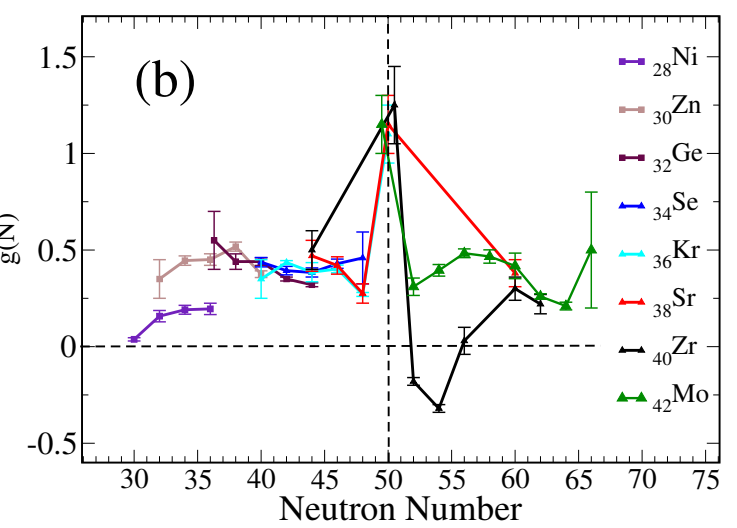

Figure 1: $g$-factor values for (a) the region around $N=20$ and $N=28$, and (b) for the region around $N=50$ and $N=28$. Experimental results before 2011 have been taken from Ref. [1]. 
Magnetic moments measurements with the TF technique, in the region around $20<N<28$ are presented in Fig. 1 (a). For values obtained before 2011 the average value from Ref. [1] is reported in this contribution. For the magic numbers at $N=20$ and $N=28$ a maximum in the positive values for the $g$ factors is observed, this result is due to the filling of the $1 d_{3 / 2}$ and $1 f_{7 / 2}$ neutron orbitals with the subsequent dominance of the proton configurations. Such behavior is also observed along the isotopic chains for the other magic numbers, see Fig. 1 (b) for $N=50$ and Fig. 2 (b) for $N=82$.

The results for nuclei in the region allow to study the behavior around the ${ }^{40} \mathrm{Ca}$ and ${ }^{48} \mathrm{Ca}$ closed-shell nuclei. For the ${ }_{18}^{40} \mathrm{Ar}$ a near-spherical shell model picture may be assumed to explain the $g\left(2_{1}^{+}\right)=-0.015(42)$ value [21], while for ${ }_{20}^{42} \mathrm{Ca}$, the isotone of ${ }^{40} \mathrm{Ar}$, it was necessary to involve significant core excitation in order to explain the vanishingly small $g\left(2_{1}^{+}\right)=+0.04(6)$ [22]. Along with the results for ${ }_{20}^{42} \mathrm{Ca}$, the positive value for ${ }_{20}^{44} \mathrm{Ca}$ of $g\left(2_{1}^{+}\right)=+0.17(3)$ is an unexpected result also [22], since a negative $g$-factor value is expected for both isotopes from two and four valence neutrons in the $f p$ shell using a ${ }_{20}^{40} \mathrm{Ca}$ as a closed-shell core. The measured $g\left(2_{1}^{+}\right)$for ${ }^{42.44} \mathrm{Ca}$ requires a significant core-excitation component in the $2_{1}^{+}$wave function. For the negative $g\left(2_{1}^{+}\right)=$ $-0.26(6)$ value for ${ }_{20}^{46} \mathrm{Ca}(f p)_{v}^{6}$ configurations dominate near the shell closure at ${ }^{48} \mathrm{Ca}$ [23]. These results allow to conclude that ${ }^{48} \mathrm{Ca}$ is a better closed-shell nucleus than ${ }^{40} \mathrm{Ca}$.

For the radioactive ${ }_{16}^{38} \mathrm{~S}$ and ${ }_{16}^{40} \mathrm{~S}$ isotopes the dominant components of the shell model wave functions are correct to give a satisfactory description of the measured $g$ factors [24].

\section{The region around $N=50$}

Measurements of nuclear magnetic moments in the region around $N=50$, using the TF technique, are presented in Fig. 1 (b). The region is of high interest due to the possibility to study the $N, Z=40$ sub-shell closure, and in fact this has motivated an important part of the measurements in the region $[25,26,27]$. Overall, the behavior for most of the $g\left(2_{1}^{+}\right)$values follows the trend around $g=Z / A$. For example, a recent experimental study of the $g$-factor values for the isotopic chain of ${ }_{32}^{70-76} \mathrm{Ge}[6],{ }^{74-82} \mathrm{Se}[28]$ and ${ }^{76-82} \mathrm{Kr}[29,27]$ found good agreement with predictions from the IBA-II model.

A very interesting case are the $g\left(2_{1}^{+}\right)$values of the $\mathrm{Zr}$ isotopes $(Z=40)$. A large positive $g\left(2_{1}^{+}\right)=+1.25(20)$ value for ${ }^{90} \mathrm{Zr}$ is produced by a predominantly $\pi\left(g_{9 / 2}\right)^{2}$ configuration, whereas the negative $g\left(2_{1}^{+}\right)=$values of ${ }^{92} \mathrm{Zr}$ and ${ }^{94} \mathrm{Zr}$ are produced by neutron excitations in the $v\left(d_{5 / 2}\right)^{2}$ and $v\left(d_{5 / 2}\right)^{4}$ respectively [26]. Values away from the $g=Z / A$ can be observed in the ${ }_{28} \mathrm{Ni}$ isotopes, are caused by an strong coupling of valence particles to an excited double-magic ${ }_{28}^{56} \mathrm{Ni}_{28}$ core [30].

\section{The region $50<N<82$ and around $N=82$}

The region for $50<N<82$ presents long isotopic chains with a collective behavior overall, as shown in Fig. 2 (a). A very interesting evolution of the magnetic moments as a function of the neutrons is presented by the ${ }_{50} \mathrm{Sn}$ chain, the $g$-factor values evolve from positive values for the lighter isotopes to negative values for the heavier ones. A qualitative analysis of this behavior is performed in Ref. [5]. The gradually filling of the set of neutron orbitals $0 g_{7 / 2}, 1 d_{5 / 2}, 1 d_{3 / 2}$, $2 s_{1 / 2}$ and $0 h_{11 / 2}$ is responsible for the evolution of the $g$-factor values along the isotopic chains. 
$\mathrm{g}\left(2_{1}^{+}\right)$factors

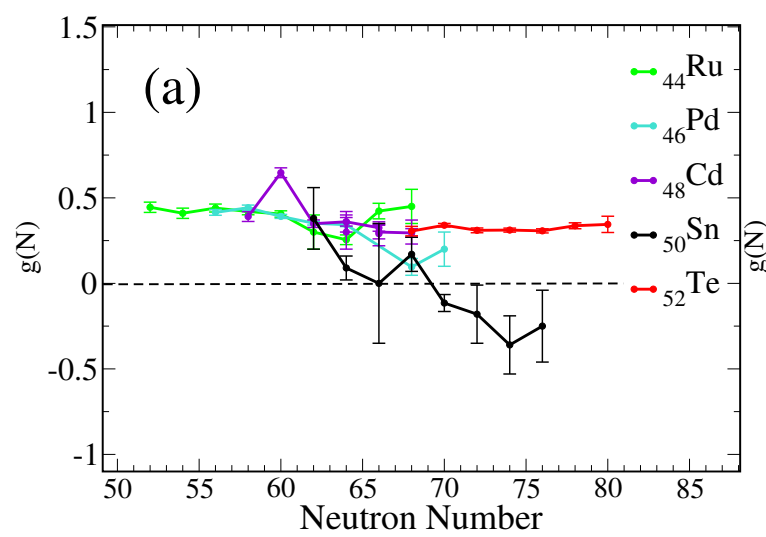

$\mathrm{g}\left(2_{1}^{+}\right)$factors

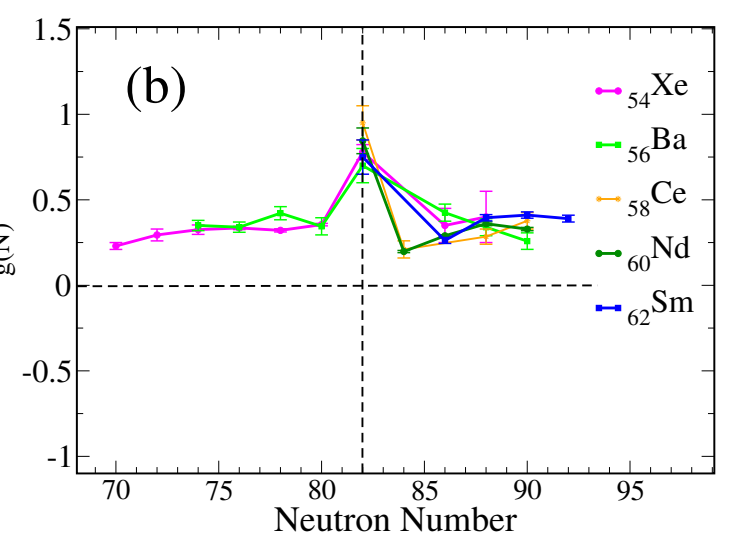

Figure 2: $g$-factor values for (a) the region $50<N<82$ using the TF technique, and (b) for the region $N=82$ using the TF technique. Experimental results before 2011 have been taken from Ref. [1].

Table 1: Empirical single-particle $g$ factors from Ref. [5] for the neutron orbitals in the $N=50-82$ major shell, and selected proton orbitals relevant for proton core excitations across $Z=50$ and resulting empirical $g$ factors of two-quasiparticle configurations with $J^{\pi}=2^{+}$.

\begin{tabular}{|c|c|c|c|}
\hline \multicolumn{2}{|c|}{ Single-particle $g$ factors } & \multicolumn{2}{|c|}{$2_{1}^{+}$state configuration } \\
\hline$\underline{\text { Orbit }}$ & $\underline{g_{\text {emp }}}$ & Configuration & $\underline{g_{e m p}}$ \\
\hline$v d_{5 / 2}$ & $\overline{-0.43}$ & $v d_{5 / 2} g_{7 / 2}$ & $\overline{+0.23}$ \\
\hline$v g_{7 / 2}$ & +0.18 & $v d_{5 / 2}^{-1} s_{1 / 2}$ & -0.20 \\
\hline$v s_{1 / 2}$ & -1.8 & $v d_{3 / 2} s_{1 / 2}$ & -0.11 \\
\hline$v d_{3 / 2}$ & +0.46 & $v g_{7 / 2}^{-1} d_{3 / 2}$ & +0.04 \\
\hline$v h_{11 / 2}$ & -0.25 & & \\
\hline$\pi g_{9 / 2}$ & +1.22 & $\pi g_{9 / 2} d_{5 / 2}$ & +1.09 \\
\hline$\pi d_{5 / 2}$ & +1.38 & $\pi g_{9 / 2} g_{7 / 2}$ & +1.34 \\
\hline$\pi g_{7 / 2}$ & +0.73 & & \\
\hline
\end{tabular}

Table 1 shows the empirical single-particle $g$ factor values, deduced from measured $g$ factors of one-quasiparticle states in odd- $A \mathrm{Sn}$ isotopes.

For the lightest $\mathrm{Sn}$ isotopes, ${ }^{112} \mathrm{Sn}$, the $g_{7 / 2}^{2}$ and the $d_{5 / 2} g_{7 / 2}$ two neutron configurations are expected to contribute to the formation of the $2_{1}^{+}$state. No notable differences exist between the $2_{1}^{+}$states of ${ }^{112} \mathrm{Sn}$ and ${ }^{114} \mathrm{Sn}$. The decrease of the $g\left(2_{1}^{+}\right)$values between ${ }^{114} \mathrm{Sn}$ and ${ }^{116} \mathrm{Sn}$ is explained by the fact that the $d_{3 / 2}$ and $s_{1 / 2}$ orbitals are already half filled, and the $d_{3 / 2} s_{1 / 2}$ configuration $(g=-0.11)$ becomes relevant. The positive value of the $g\left(2_{1}^{+}\right)$for ${ }^{118} \mathrm{Sn}$ goes off the systematics, in Ref. [31] this behavior is attributed to the influence of the $d_{3 / 2}^{2}$ neutron configuration ( $g=+0.46$ in Table 1$)$ that has half the occupancy according to the results from Ref. [32]. For the remaining heavier ${ }^{120-126} \mathrm{Sn}$ isotopes, the $h_{11 / 2}$ orbital is being filled and plays an important role 
in the description of the configuration of the $2_{1}^{+}$states $[5,9]$.

5. The region with $N>82$
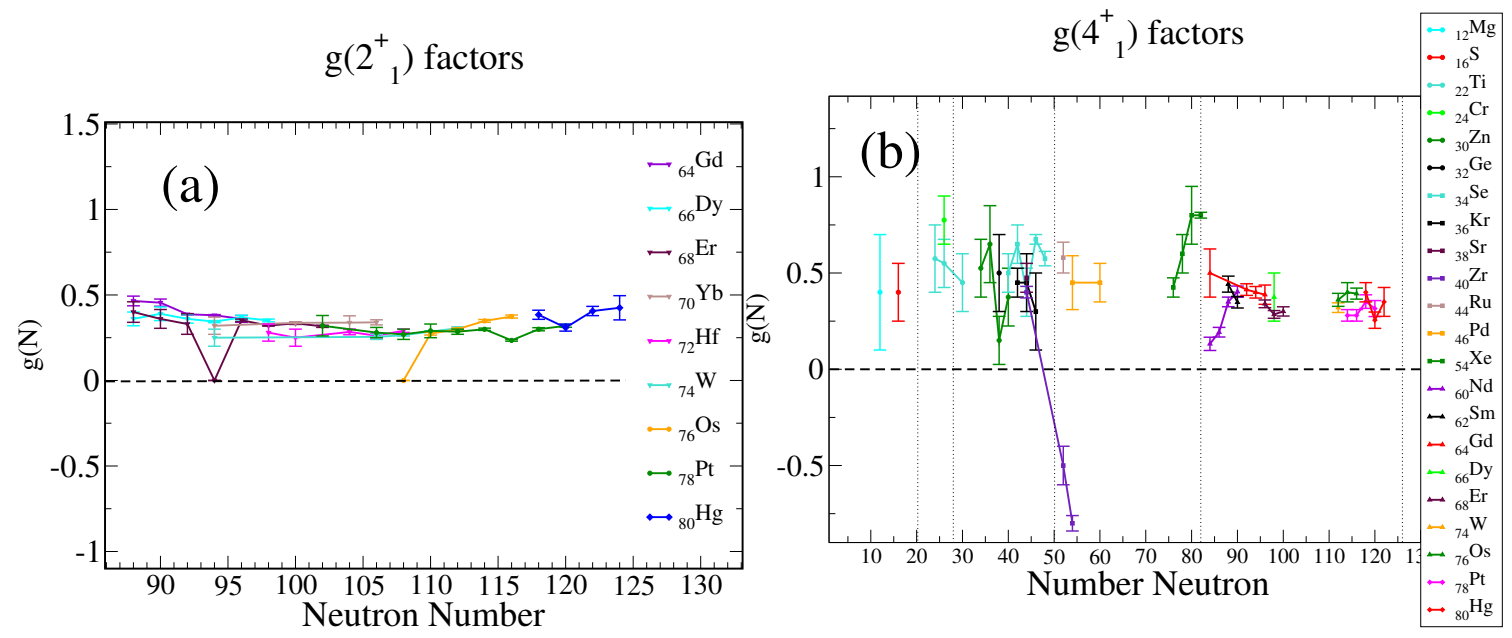

Figure 3: $g$-factor values for (a) the region $82<N<126$ measured using the TF technique, and (b) for the $g$-factor values of the $4_{1}^{+}$states in even-even nuclei measured using the TF technique. Vertical dotted lines correspond to the magic numbers for neutrons. Experimental results before 2011 have been taken from Ref. [1].

The onset of collectivity, for isotopes with $82<N<126$, is clearly seen in Fig. 3 (a), where $g$-factor values for isotopes with $82<N<126$ are presented. The influence of the $\pi\left(g_{7 / 2}\right)$ and $v\left(f_{7 / 2}\right)$ orbitals are important to explain magnetic moments around the ${ }^{132} \mathrm{Sn}$ [33]. Deviation from the $g \sim Z / A$ value have been explained with the help of pairing, single-particle contribution or subshell closure at $Z=64$ [34].

An interesting feature for the region is the possibility to easily measure $g$ factors for states with $J>2$. This is possible due to two main reasons: firstly, the nuclear level density of the nuclei in the region that determines the possibility to make use of Coulomb excitation reactions [34], in some cases the ratios $g\left(4_{1}^{+}\right) / g\left(2_{1}^{+}\right)$and $g\left(6_{1}^{+}\right) / g\left(2_{1}^{+}\right)$are reported [35, 36] instead of single values of $g\left(2_{1}^{+}\right)$. Secondly, the possible use of Fusion-Evaporation reactions (FE) to populate collective structures. The main limitation for the use of FE reactions is that the time history of the de-exciting nuclei is not always well established, this obstacle sets limits to the information of the magnetic interaction between the excited states and the host ferromagnetic [37, 38], some works reports average $g$-factor values for deformed structures in specific isotopes $[37,39]$ and quasicontinuum structures [40].

\section{6. $g$-factor values for $J>2$ states}

In Fig. 3 the $g$-factor values of the $4_{1}^{+}$states measured using the TF technique are presented. The small number of $g$ factor measured, when compared to the number of $g\left(2_{1}^{+}\right)$, is due to the 
experimental difficulties to populate and simultaneously measure the precession of the states with $J \geq 4$ in certain mass regions. As has been presented in previous sections of this contribution, three reactions have been commonly utilized to populated such states, Coulomb excitation, $\alpha$ transfer, and fusion-evaporation. Coulomb excitation provides the most reliable result and fine results for states close to the ground state. while fusion-evaporation reactions can populate highspin states, the measured precession of a given state maybe contaminated by the precession from higher-feeding states.

Recent studies using $\alpha$-transfer reactions and Coulomb excitation in the $A \sim 100$ region have pointed out the importance of measuring $g$-factor values for $J>2$ states. Two interesting cases will be presented here, the negative $g\left(4_{1}^{+}\right)$value of ${ }_{38}^{86} \mathrm{Sr}$ and the $g\left(4_{1}^{+}\right)$values for ${ }^{100} \mathrm{Pd}$ and ${ }^{96} \mathrm{Ru}$.

\subsection{The negative $g\left(4_{1}^{+}\right)$value of ${ }_{38}^{86} \mathrm{Sr}$}

A surprisingly negative $g\left(4_{1}^{+}\right)=-0.68(49)$ Value for ${ }^{86} \mathrm{Sr}$ Has been recently reported in Ref. [15], in contrast with the positive values of $g\left(2_{1}^{+}\right)=+0.285(14)$ and $g\left(2_{2}^{+}\right)=+0.40(16)$ for the ${ }^{86} \mathrm{Sr}$ isotope, and $g\left({ }^{88} \mathrm{Zr} ; 4_{1}^{+}\right)=+0.65(18)$. Shell model calculations using the JUN45 [19] and JJ4B [18] interactions predict a small-negative value of $g\left(4_{1}^{+}\right)_{J U N 45}=-0.07$ and a positive $g\left(4_{1}^{+}\right)_{J J 4 B}=+0.22$, using a valence space composed by the single-particle orbitals $1 p_{3 / 2}, 0 f_{5 / 2}$, $1 p_{1 / 2}$, and $0 g_{9 / 2}$, for both protons and neutrons.

In the considered space only three orbitals have negative single-particle Schmidt $g$ factor values, $g\left(p_{1 / 2}\right)_{\pi}=-0.529, g\left(g_{9 / 2}\right)_{v}=-0.425$ and $g\left(p_{3 / 2}\right)_{v}=-1.275$. It is not easy to figure out a possible configuration, that will form a $4^{+}$state using only a proton configuration, while for neutrons the configurations $g\left(g_{9 / 2}\right)_{v}^{2}$ or $g\left(p_{3 / 2}, f_{5 / 2}\right)_{v}^{2}$ can form a $4^{+}$. Future Shell-Model studies should clarify the origin of this value, and also need a better experimental value.

6.2 The trend of the $g\left(2_{1}^{+}\right)$and $g\left(4_{1}^{+}\right)$values for ${ }^{100} \mathbf{P d}$ and ${ }^{96} \mathbf{R u}$

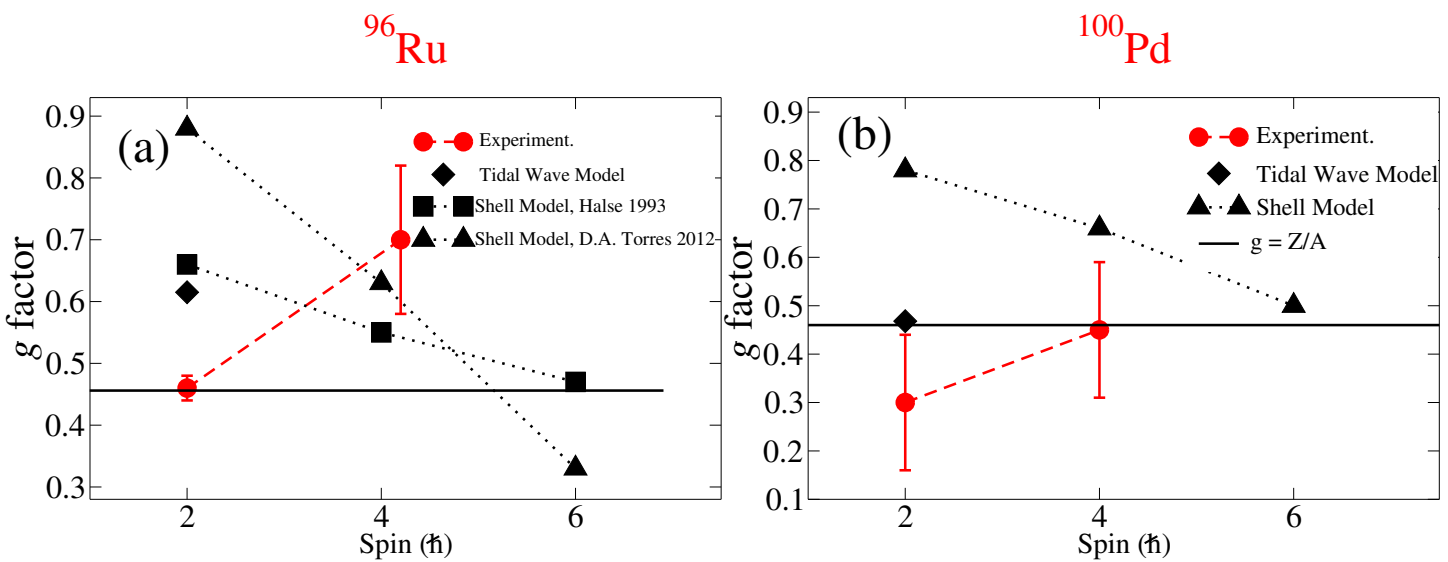

Figure 4: Trend of the experimental and theoretical $g$-factor values for (a) ${ }^{96} \mathrm{Ru}$ and (b) ${ }^{100} \mathrm{Pd}$.

The study of trends of the $g$-factor values as a function of the spin of the states provides an interesting test to the microscopic predictions of nuclear models. Recent results in ${ }^{100} \mathrm{Pd}$ [11] and ${ }^{96} \mathrm{Ru}[41]$ present a clear example of this, in Fig. 4 the experimental results are compared with Shell 
Model calculations presented in their respective references. In both case the experimental values do not follow the theoretical predictions. Future work to clarify the differences in these trends is needed.

\section{Summary and future}

In this contribution an overview of the systematic of the nuclear $g$ factors for several isotopic chains was presented. For nuclei with a neutron number around a magic number a clear singleparticle behavior is present, and for nuclei in-between magic numbers a trend following the $g=Z / A$ prediction from collective models is followed. For isotopic chains that are out of the latter general trend, the influence of $j$ orbitals explain the behavior of $g$-factor values for the isotopic chains; this is the case of the $\pi\left(g_{9 / 2}\right)$ for the $\mathrm{Sr}$ isotopes and the $v\left(h_{11 / 2}\right)$ for the Sn isotopes.

The investigation of the softness of the ${ }^{88} \mathrm{Sr}$ and ${ }^{90} \mathrm{Zr}$ have stimulated recent studies at the extremes of the $\operatorname{Sr}(Z=38)$ isotopic chain, for the radioactive ${ }^{82} \mathrm{Sr}$ and ${ }^{90} \mathrm{Sr}$, specifically, have been carried out using $\alpha$-transfer reaction from $\mathrm{Kr}$ isotopes [42].

The investigation of trends of magnetic moments as a function of the nuclear spin provides a test of the microscopic prediction of the different models. Future experimental studies should exploit:

- the use of $\alpha$-transfer reactions to populate medium spin states. In recent years, the use of $\alpha$-transfer reactions has become an active area of research which allows the production of states in radioactive species that otherwise will only be available in future radioactive beam facilities. A hindrance to the use of the $\alpha$-transfer mechanism is that the alignment of the nuclear spin, necessary to observe a precession of the magnetic moment in an external or a hyperfine magnetic field, is much reduced compared to that observed in experiments using traditional Coulomb excitation.

Future studies must focus on improving the precision of the experimental results for $\alpha$ transfer reactions. Such studies need a detailed characterization of the reaction and the angular particle- $\gamma$ correlations. The final goal is the design of new experimental setups with the optimization of the position of the charged-particle detector, and the use of highly segmented $\Delta E-E$ detectors.

- the use of radioactive beams. Future studies of $g$ factors for neutron-rich nuclei will depend on the development of experimental programs to measure in radioactive beam facilities. Some studies have been carried out recently $[9,10]$. The use of Coulomb excitation reactions will be the best way to initiate future studies in this field. The main challenges arise from the available beam intensities.

- The study of the ion-solid interaction and the development of a new generation of target. The study of the interaction ion-solid is pivotal to develop a new generation of targets for $g$ factor measurements. To date, the use of several parametrizations, to obtain values of the hyperfine field utilized in TF experiments [3, 2], have a common linear dependence with the number $Z$ of the ions but differ in their explicitly velocity dependence. The use of only 
one parametrization deduced from first principles is desirable as one of the goals of future studies.

\section{Acknowledgments}

The authors are indebted to G.J. Kumbartzki, N. Koller, Y.Y. Sharon, F. Nowacki and L.F. Cristancho for their comments to the present work. The authors acknowledges financial support from Universidad Nacional de Colombia and the Faculty of Science.

\section{References}

[1] N.J. Stone. Table of nuclear magnetic dipole and electric quadrupole moments. Technical report, Research sponsored by the IAEA Nuclear Data Section, Vienna International Centre, 1400 Vienna, Austria, April 2011.

[2] K. H. Speidel, O. Kenn, and F. Nowacki. Magnetic moments and nuclear structure. Prog.Part.Nucl.Phys., 49:91, 2002.

[3] N. Benczer-Koller and G.J. Kumbartzki. Magnetic moments of short-lived excited nuclear states: measurements and challenges. Journal of Physics G: Nuclear and Particle Physics, 34(9):R321, 2007.

[4] A.E. Stuchbery. Simple structures in complex nuclei versus complex structures in simple nuclei: a nuclear moments perspective. Journal of Physics: Conference Series, 366(1):012042, 2012.

[5] J. Walker et al. Magnetic moments of the first excited $2^{+}$states in the semi-magic ${ }^{112,114,116,122,124} \mathrm{Sn}$ isotopes. Phys. Rev. C, 84:014319, Jul 2011.

[6] G. Gürdal et al. Measurements of $g\left(4_{1}^{+}, 2_{2}^{+}\right)$in ${ }^{70,72,74,76} \mathrm{Ge}$ : Systematics of low-lying structures in $30 \leq \mathrm{Z} \leq 40$ and $30 \leq \mathrm{N} \leq 50$ nuclei. Phys. Rev. C, 88:014301, Jul 2013.

[7] N.J. Stone et al. First nuclear moment measurement with radioactive beams by the recoil-in-vacuum technique: The $g$ factor of the $2_{1}^{+}$state in ${ }^{132}$ Te. Phys. Rev. Lett., 94:192501, May 2005.

[8] N. Benczer-Koller et al. Measurement of $\mathrm{g}$ factors of excited states in radioactive beams by the transient field technique: ${ }^{132}$ Te. Physics Letters B, 664(4-5):241 - 245, 2008.

[9] G.J. Kumbartzki et al. Transient field $g$ factor and mean-life measurements with a rare isotope beam of ${ }^{126}$ Sn. Phys. Rev. C, 86:034319, Sep 2012.

[10] E. Fiori et al. First $g\left(2^{+}\right)$measurement on neutron-rich ${ }^{72} \mathrm{Zn}$, and the high-velocity transient field technique for radioactive heavy-ion beams. Phys. Rev. C, 85:034334, Mar 2012.

[11] D.A. Torres et al. First $g$-factor measurements of the $2_{1}^{+}$and the $4_{1}^{+}$states of radioactive ${ }^{100} \mathrm{Pd}$. Phys. Rev. C, 84:044327, Oct 2011.

[12] S. Schielke et al. First measurement and shell model interpretation of the $g$ factor of the $2_{1}^{+}$state in self-conjugate radioactive ${ }^{44}$ Ti. Physics Letters B, 567(3-4):153 - 158, 2003.

[13] K.-H. Speidel et al. Experimental g factors and B(E2) values in Ar isotopes: Crossing the N=20 semi-magic divide. Physics Letters B, 632(2-3):207 - 211, 2006.

[14] J. Leske et al. Nuclear structure of the first $2+$ state in radioactive ${ }^{68} \mathrm{Ge}$ based on $g$ factor and lifetime measurements. Phys. Rev. C, 71(4):044316, Apr 2005.

[15] G.J. Kumbartzki et al. Structure of the $\mathrm{Sr}-\mathrm{Zr}$ isotopes near and at the magic $N=50$ shell from $g$-factor and lifetime measurements in ${ }_{40}^{88} \mathrm{Zr}$ and ${ }^{84,86,88}$ Sr. Phys. Rev. C, 85:044322, Apr 2012. 
[16] E. Caurier, G. Martínez-Pinedo, F. Nowacki, A. Poves, and A. P. Zuker. The shell model as a unified view of nuclear structure. Rev. Mod. Phys., 77:427-488, Jun 2005.

[17] Morten Hjorth-Jensen, Thomas T. S. Kuo, and Eivind Osnes. Realistic effective interactions for nuclear systems. Physics Reports, 261(3-4):125 - 270, 1995.

[18] A.F. Lisetskiy et al. New $T=1$ effective interactions for $f_{5 / 2} p_{3 / 2} p_{1 / 2} g_{9 / 2}$ the model space: Implications for valence-mirror symmetry and seniority isomers. Phys. Rev. C, 70:044314, Oct 2004.

[19] M. Honma, T. Otsuka, T. Mizusaki, and M. Hjorth-Jensen. New effective interaction for $f p g$-shell nuclei. Phys. Rev. C, 80:064323, Dec 2009.

[20] S. M. Lenzi, F. Nowacki, A. Poves, and K. Sieja. Island of inversion around ${ }^{64}$ Cr. Phys. Rev. C, 82:054301, Nov 2010.

[21] E.A. Stefanova et al. Near spherical shell-model structure of the $2_{1}^{+}$state in ${ }^{40} \mathrm{Ar}$ from $g$-factor measurements. 72:014309, Jul 2005.

[22] S. Schielke et al. Evidence for ${ }^{40} \mathrm{Ca}$ core excitation from $g$ factor and B(E2) measurements on the $2_{1}^{+}$ states of ${ }^{42,44}$ Ca. Phys.Lett. B, 571:29, 2003.

[23] M. J. Taylor et al. Shell model configurations in the $2_{1}^{+}$state in ${ }^{46} \mathrm{Ca}$ from a $g$-factor measurement. Phys.Lett. B, 605:265, 2005.

[24] A.E. Stuchbery et al. Shell structure underlying the evolution of quadrupole collectivity in ${ }^{38} \mathrm{~S}$ and ${ }^{40} \mathrm{~S}$ probed by transient-field $g$-factor measurements on fast radioactive beams. Phys. Rev. C, 74:054307, Nov 2006.

[25] V. Werner. Evidence for the microscopic formation of mixed-symmetry states from magnetic moment measurements. Phys. Rev. C, 78:031301, Sep 2008.

[26] A. E. Stuchbery, N. Benczer-Koller, G. Kumbartzki, and T. J. Mertzimekis. Gyromagnetic ratios and octupole collectivity in the structure of the ${ }^{96-96} \mathrm{Zr}$ isotopes. Phys. Rev. C, 69:044302, Apr 2004.

[27] T.J. Mertzimekis. First measurements of $g$ factors in the even $\mathrm{Kr}$ isotopes. Phys. Rev. C, 64(2):024314, Jul 2001.

[28] K.-H. Speidel et al. Shell closure effects in the stable ${ }^{74-82}$ Se isotopes from magnetic moment measurements using projectile excitation and the transient field technique. Phys. Rev. C, 57(5):2181-2188, May 1998.

[29] G. Kumbartzki et al. First $g$ factor measurement using a radioactive ${ }^{76} \mathrm{Kr}$ beam. Physics Letters B, 591(3-4):213 - 219, 2004.

[30] O. Kenn. Striking harmony between the nuclear shell model and new experimental $g$ factors and B(E2) values of even Ni isotopes. Phys. Rev. C, 63:021302, Dec 2000.

[31] M.C. East et al. $g$ factors in ${ }^{116,118,120} \mathrm{Sn}$ : Sensitivity to configurations near the fermi surface. Physics Letters B, 665(4):147 - 151, 2008.

[32] M Anguiano, J.L Egido, and L.M Robledo. Mean-field based approaches to pairing correlations in atomic nuclei. Physics Letters B, 545(1-2):62 - 72, 2002.

[33] A.E. Stuchbery et al. Electromagnetic properties of the $2_{1}^{+}$state in ${ }^{134} \mathrm{Te}$ : Influence of core excitation on single-particle orbits beyond ${ }^{132}$ Sn. Phys. Rev. C, 88:051304, Nov 2013.

[34] J. Holden et al. Evidence for $2 f_{7 / 2}$ neutron strength in the low energy structure of ${ }^{144,146,148,150} \mathrm{Nd}$ isotopes. Phys.Lett., 493B:7, 2000. 
[35] N.A. Matt et al. Competition between single-particle and collective excitations in $N=88$ nuclei: Measurement of $g\left(4_{1}^{+}\right) / g\left(2_{1}^{+}\right)$in ${ }^{152}$ Gd. Phys. Rev. C, 59:665-668, Feb 1999.

[36] T. Vass et al. Measurements of magnetic moments in ${ }^{150}$ Sm. Phys. Rev. C, 48:2640-2647, Dec 1993.

[37] M. Hass et al. Decay history and magnetic moments at high spin in ${ }^{152}$ Dy. Phys.Rev., C44:1397, 1991.

[38] L. Weissman et al. Time-decay history of normal-deformation high-spin states in ${ }^{193,194} \mathrm{Hg}$. Nucl.Phys., A645:191, 1999.

[39] R.H. Mayer et al. First direct measurements of $g$ factors of the three superdeformed bands of ${ }^{194} \mathrm{Hg}$. Phys. Rev. C, 58:R2640-R2643, Nov 1998.

[40] L. Weissman et al. Single particle signatures in high-spin, quasicontinuum states in ${ }^{193,194} \mathrm{Hg}$ from g-factor measurements. Phys.Lett., 446B:22, 1999.

[41] D.A. Torres et al. Measurement of the ${ }^{96} \mathrm{Ru} g\left(4_{1}^{+}\right)$factor and its nuclear structure interpretation. Phys. Rev. C, 85:017305, Jan 2012.

[42] G.J. Kumbartzki. Transition from collectivity to single-particle degrees of freedom from magnetic moment measurements on ${ }_{38}^{82} \mathrm{Sr}_{44}$ and ${ }_{38}^{90} \mathrm{Sr}_{52}$. Phys. Rev. C, 89:064305, Jun 2014. 